\title{
Evaluación de la aplicación de pruebas de diagnóstico rápido para malaria como parte de una estrategia integral para su control en Colombia
}

\author{
Nohora Marcela Mendoza1, Ángel Martín Rosas¹, Javier Darío Burgos² \\ 1 Proyecto Control de la malaria en zonas fronterizas de la Región Andina: un enfoque comunitario, PAMAFRO, \\ Organismo Andino de Salud, Lima, Perú \\ 2 Instituto de Estudios Ambientales, Universidad Nacional de Colombia, Bogotá, D.C., Colombia
}

Introducción. El Organismo Andino de la Salud coordinó y evaluó la estrategia de aplicación de pruebas de diagnóstico rápido de malaria para fortalecer el diagnóstico de esta enfermedad en diez departamentos de Colombia.

Objetivo. Evaluar el ciclo de implementación, impacto, resultado, uso y cobertura de la aplicación de las pruebas de diagnóstico rápido en el ámbito del proyecto Control de la malaria en zonas fronterizas de la Región Andina: un enfoque comunitario (PAMAFRO), como parte de una estrategia integral para el control de la malaria.

Materiales y métodos. En un estudio descriptivo retrospectivo, se evaluó el ciclo de implementación del uso de las pruebas de diagnóstico rápido, primera etapa, y los indicadores de resultado, uso y cobertura de la estrategia, segunda etapa, durante el periodo de octubre de 2007 a julio de 2008. Se aplicó el análisis multicriterio, método de puntaje para determinar las variables críticas.

Resultados. El cumplimiento del ciclo de implementación fue de $71 \%$ y la planeación fue el componente más débil del ciclo, con un cumplimiento del $50 \%$. Se determinaron como variables críticas y con bajo cumplimiento las siguientes: estudio de las necesidades de pruebas de diagnóstico rápido en el país (50\%), estudio de necesidades en los departamentos (50\%), distribución de las pruebas según las necesidades (50\%), evaluación del desempeño de los trabajadores de salud (50\%), cumplimiento de directrices sobre temperatura y humedad de las pruebas en el nivel departamental (50\%), logística (50\%) y supervisión ( $25 \%)$.

Conclusiones. Es importante que en futuras estrategias de aplicación de pruebas de diagnóstico rápido en el país, se fortalezcan las variables críticas de bajo cumplimiento encontradas en el presente estudio.

Palabras clave: malaria/diagnóstico, paludismo, control de enfermedades transmisibles, implementación de plan de salud, Colombia.

\section{Evaluation of rapid diagnostic tests for malaria in Colombia as an integral part of the disease control strategy}

Introduction. The Andean Health Organization has been responsible for the coordination and evaluation of the malaria rapid diagnostic test strategy. This undertaking was organized to strengthen the malaria diagnostic capacity in ten provinces of Colombia.

Objective. The implementation cycle of malaria rapid diagnostic tests was evaluated, along with its impact, performance, usage and coverage under the project "Malaria control in bordering areas of the Andean Region: a community cpproach (PAMAFRO), as an integral part of the malaria control strategy.

Materials and methods. A descriptive retrospective study was organized in two stages. The first stage was an evaluation of the implementation of the rapid diagnostic test cycle. The second stage evaluated indicators of impact, performance, usage and coverage of this strategy. These evaluations were conducted from October 2007 to July 2008 in 10 Andean provinces of Colombia. A multi-criteria scoring method was applied to determine the critical variables.

Results. The compliance in the implementation cycle for rapid diagnostic tests was $71 \%$. Planning was the weakest component of the cycle with $50 \%$ of the goals accomplished. The critical variables with low compliance were as follows: study of rapid diagnostic test needs in the country (50\%), study of rapid diagnostic test needs in each province (50\%), rapid diagnostic test distribution according to needs $(50 \%)$, assessment of health workers performance $(50 \%)$, compliance with temperature and humidity requirements for storage of the rapid diagnostic tests at the provincial level $(50 \%)$, logistics $(67 \%)$ and supervision $(25 \%)$. 
Conclusion. Implementation strategies are important to strengthen the critical variables found asociated with low compliance.

Key words: Malaria/diagnosis, communicable disease control, health plan implementation, Colombia.

La implementación y el uso sostenido del diagnóstico parasitario temprano y el tratamiento oportuno y eficaz cobran importancia en la atención del paciente con malaria porque reducen la mortalidad y la morbilidad por la enfermedad (1). Por otra parte, el diagnóstico parasitario posibilita la diferenciación de la malaria de otras infecciones febriles con el fin de orientar una adecuada conducta médica, hacer uso racional de los medicamentos y contar con una estimación epidemiológica precisa de la enfermedad $(2,3)$.

La confirmación diagnóstica rutinaria puede hacerse tanto por la visualización del parásito como por la detección de sus proteínas (antígenos) mediante el uso de pruebas de diagnóstico rápido. Sin embargo, una prueba de diagnóstico rápido no reemplaza el diagnóstico por gota gruesa, debido a las ventajas que ofrece este último método $(4,5)$.

La Organización Panamericana de la Salud (OPS) ha dado directrices para contar con buenas prácticas en todo el ciclo de implementación de pruebas de diagnóstico rápido, abarcando la identificación del área, la selección de la prueba, la implementación y evaluación del uso (figura 1). De igual manera, la OPS ha impartido recomendaciones sobre condiciones ambientales durante el transporte, almacenamiento y uso de las pruebas, y lineamientos para estandarizar los contenidos de capacitación del personal encargado del diagnóstico y para el control de calidad que debe aplicarse a la adquisiciones, al mantenimiento de las pruebas durante su vida útil y a las actividades realizadas por el personal responsable de prestar el servicio de diagnóstico $(6,7)$.

Teniendo presente la anterior información, el proyecto "Control de la malaria en las zonas fronterizas de la Región Andina: un enfoque comunitario (PAMAFRO)" determinó las condiciones de adquisición y aplicación de las pruebas de diagnóstico rápido en Colombia, con el fin de fortalecer el diagnóstico de la enfermedad en

Correspondencia:

Nohora Marcela Mendoza, Organismo Andino de Salud, Calle 30A № 6-22, oficina 604, Bogotá, D.C., Colombia

Teléfono: (571) 609 0282; fax: (571) 6793992

marcemendoza07@gmail.com

Recibido: 09/02/10; aceptado:28/09/10 municipios con transmisión de malaria y con debilidades en la red de diagnóstico microscópico.

Debido a que a que la mayoría de publicaciones sobre pruebas de diagnóstico rápido están enfocadas a estudiar su capacidad diagnóstica, el presente trabajo evaluó el ciclo de implementación de las pruebas, como parte de una estrategia integral y como producto del trabajo realizado dentro del proyecto PAMAFRO.

\section{Materiales y métodos}

\section{Tipo de estudio}

Se trató de un estudio descriptivo retrospectivo de la aplicación de las pruebas de diagnóstico rápido para malaria.

\section{Lugar y período de estudio}

Se llevó a cabo en los departamentos de Amazonas, Arauca, Boyacá, Cesar, Guainía, La Guajira, Nariño, Norte de Santander, Putumayo y Vichada, desde octubre de 2007 hasta julio de 2008.

\section{Metodología}

La evaluación de la aplicación de las pruebas de diagnóstico rápido para malaria en Colombia utilizó las pruebas OptiMal $\AA^{\circledR}$, que se aplicaron en municipios y localidades determinadas por el proyecto PAMAFRO y por los coordinadores del Programa de Enfermedades Transmitidas por Vectores (ETV) en cada departamento. La evaluación de la aplicación de la prueba de diagnóstico rápido constó de dos etapas: la evaluación del cumplimiento del ciclo de implementación y la evaluación de indicadores de resultado, uso y cobertura de la estrategia de implementación. Este trabajo no evaluó la eficacia.

Evaluación del cumplimiento del ciclo de implementación de las pruebas de diagnóstico rápido (primera etapa). Se hizo tomando como base el documento titulado "Investigación operacional sobre la implementación del uso de pruebas rápidas de diagnóstico de malaria" propuesto en la reunión de la Red Amazónica de Vigilancia de la Resistencia a los Antipalúdicos (RAVREDA), Iniciativa Amazónica de Malaria (AMI), realizada en Guayaquil en 2005 (8).

Para la evaluación del cumplimiento del ciclo de implementación de las pruebas de diagnóstico 
rápido, el proyecto PAMAFRO aplicó una metodología de análisis multicriterio de la siguiente manera: se diseñó una matriz que incluyó componentes, temas, variables e indicadores (cuadro 1). El ciclo de implementación se enmarcó en tres componentes: planeación, adquisición y garantía de la calidad. Los componentes se subdividieron en los siguientes temas: al componente de planeación le correspondió el tema de identificación de áreas; al de adquisición, el de selección de las pruebas de diagnóstico rápido, y al de garantía de calidad le correspondieron los temas de implementación y evaluación (8).

A cada tema se le asignaron variables y estas últimas se midieron con indicadores, los cuales se diseñaron de acuerdo con la información disponible dentro de la estrategia de aplicación de pruebas de diagnóstico rápido planteada por el proyecto PAMAFRO, Colombia. Para cada indicador se definieron niveles de satisfacción utilizando escalas binarias o $\mathrm{n}$-arias. El valor ponderado, entendido como la importancia de la variable dentro del ciclo, se obtuvo mediante consulta a expertos sobre malaria en Colombia. Mediante la multiplicación del nivel de satisfacción por el valor ponderado, se obtuvo la puntuación que determinó las variables críticas cuando se obtuvo un valor de 5 puntos o más $(9,10)$. Las últimas dos columnas del cuadro 1 presentan la ponderación y el intervalo de la puntuación para cada variable, respectivamente.

Cada indicador se evaluó mediante la revisión y el análisis de la información recolectada en la estrategia de aplicación de pruebas de diagnóstico rápido planteada por el proyecto PAMAFRO, Colombia. Las fuentes de información fueron informes y bases de datos reportados al proyecto, por parte de los departamentos intervenidos, el Ministerio de la Protección Social, el Instituto Nacional de Salud y el nivel central del proyecto PAMAFRO, que forman parte de la documentación de este proyecto. Además, en el componente "Garantía de la calidad", se incluyeron dos tipos de encuestas para la recolección de información prospectiva: encuestas de pregunta abierta con la finalidad de recabar información complementaria desde las Secretarías Departamentales de Salud y encuestas de percepción de los trabajadores de salud frente a las pruebas de diagnóstico rápido.

Teniendo en cuenta el diseño de la matriz del ciclo de implementación del proyecto PAMAFRO Colombia, los cálculos realizados para el cumplimiento resultaron de la división de la puntuación obtenida para cada variable crítica entre la puntuación máxima de la variable, por 100. El cumplimiento de cada variable, expresado en forma de porcentaje, resultó del promedio simple de los porcentajes de cumplimiento de los indicadores para cada variable $y$, a su vez, el cumplimiento de los temas evaluados se obtuvo del promedio simple de los porcentajes de cumplimiento de las variables definidas. De igual forma, el cumplimiento de cada componente del ciclo de implementación resultó del promedio simple de los porcentajes de cumplimiento de los temas para cada componente. Finalmente, el cumplimiento del ciclo de implementación de la estrategia en Colombia resultó del promedio simple de los porcentajes de los componentes (cuadro 2). En todos los casos se consideró como aceptable un cumplimiento de $70 \%$ o mayor, valor que se determinó por satisfacer en mayor medida el cumplimiento de las metas generales del proyecto PAMAFRO Colombia.

El análisis estadístico incluyó el análisis multicriterio con el método del puntaje, para determinar las variables críticas de una manera rápida y sencilla $(9,10)$.

Determinación de indicadores de resultado, uso y cobertura de la estrategia de implementación de las pruebas de diagnóstico rápido (segunda etapa). Se determinaron los indicadores que se listan a continuación.

- Indicadores de resultado

Nombre del indicador. Casos de malaria determinados mediante pruebas de diagnóstico rápido en Colombia.

Definición del indicador: expresa el total de enfermos atendidos con pruebas de diagnóstico rápido en los departamentos objeto del proyecto durante el período evaluado y en comparación con años anteriores a partir del 2002.

Nombre del indicador. Porcentaje de pacientes con malaria determinados con pruebas de diagnóstico rápido y que recibieron tratamiento antipalúdico durante el período de intervención.

Definición del indicador: expresa la proporción de pacientes positivos para malaria y cuyo diagnóstico se hizo con estas pruebas y que fueron tratados con medicamento antipalúdico.

Nombre del indicador. Porcentaje de epidemias o brotes intervenidos con pruebas de diagnóstico rápido en Colombia durante la intervención. 
Cuadro 1. Temas, variables, indicadores y niveles de satisfacción del ciclo de implementación.

\begin{tabular}{|c|c|c|c|c|c|c|}
\hline Componente & Tema & Variable & Indicador & $\begin{array}{l}\text { Niveles de } \\
\text { satisfacción }\end{array}$ & Ponderación & $\begin{array}{c}\text { Intervalo } \\
\text { de la variable }\end{array}$ \\
\hline \multirow[t]{4}{*}{ Planeación } & \multirow[t]{4}{*}{$\begin{array}{l}\text { Identificación de } \\
\text { áreas }\end{array}$} & $\begin{array}{l}\text { Estudio de necesidades } \\
\text { en el país }\end{array}$ & $\begin{array}{l}\text { ¿Hubo consulta adecuada } \\
\text { del nivel central sobre las } \\
\text { necesidades de PDR para } \\
\text { Colombia? }\end{array}$ & $\begin{array}{l}\text { Nulo (0), parcial (1), } \\
\text { total (2) }\end{array}$ & 5 & $(0-10)$ \\
\hline & & $\begin{array}{l}\text { Estudio de necesidades } \\
\text { en los departamentos }\end{array}$ & $\begin{array}{l}\text { ¿Hubo consulta adecuada } \\
\text { de las necesidades de PDR } \\
\text { en el nivel departamental? }\end{array}$ & $\begin{array}{l}\text { Nulo }(0) \text {, parcial }(1) \text {, } \\
\text { total }(2)\end{array}$ & 5 & $(0-10)$ \\
\hline & & $\begin{array}{l}\text { Estudios de costo-efectividad } \\
\text { para la aplicación de las PDR }\end{array}$ & $\begin{array}{l}\text { ¿Existieron estudios de } \\
\text { costo-efectividad para } \\
\text { decidir la aplicación de } \\
\text { las PDR en el país? }\end{array}$ & Sí (1), no (0) & 3 & $(0-3)$ \\
\hline & & $\begin{array}{l}\text { Diseño de directrices a los } \\
\text { departamentos }\end{array}$ & $\begin{array}{l}\text { ¿Se diseñaron directrices } \\
\text { escritas para la selección } \\
\text { del área de aplicación o } \\
\text { situación en la que debía } \\
\text { aplicarse la PDR } \\
\text { por departamento? }\end{array}$ & Sí (1), no (0) & 5 & $(0-5)$ \\
\hline Total tema & \multicolumn{6}{|c|}{$\begin{array}{l}\text { A partir del producto del nivel de satisfacción y la ponderación para cada indicador, se calcula el porcentaje de cumplimiento dividiendo el } \\
\text { valor obtenido por el valor máximo del intervalo de la variable y multiplicándolo por } 100 \text {. El porcentaje de cumplimiento corresponde al mismo } \\
\text { de la variable. Se promedia el cumplimiento de cada variable para determinar el del tema general. Se estimó como cumplimiento adecuado } \\
\text { un porcentaje } \geq 70 \% \text {. }\end{array}$} \\
\hline $\begin{array}{l}\text { Total } \\
\text { componente }\end{array}$ & \multicolumn{6}{|c|}{ El porcentaje de cumplimiento para este componente corresponde al mismo valor del tema. } \\
\hline \multirow[t]{6}{*}{ Adquisición } & \multirow[t]{6}{*}{$\begin{array}{l}\text { Selección de } \\
\text { la PDR }\end{array}$} & $\begin{array}{l}\text { Capacidad para diferenciar } \\
\text { infección por } P \text {. falciparum } \\
\text { de otras especies } \\
\text { (antígenos blanco) }\end{array}$ & $\begin{array}{l}\text { ¿La prueba contó con } \\
\text { antígeno panpalúdico y } \\
\text { antígeno específico } \\
\text { para } P \text {. falciparum? }\end{array}$ & Sí (1), no (0) & 5 & $(0-5)$ \\
\hline & & Costo de la prueba & $\begin{array}{l}¿ E \text { El costo fue inferior } \\
\text { a US } \$ 1,5 ?\end{array}$ & Sí (1), no (0) & 3 & $(0-3)$ \\
\hline & & $\begin{array}{l}\text { Capacidad para realizar los } \\
\text { controles al tratamiento }\end{array}$ & $\begin{array}{l}\text { ¿El antígeno decrece } \\
\text { proporcionalmente con la } \\
\text { parasitemia? }\end{array}$ & Sí (1), no (0) & 3 & $(0-3)$ \\
\hline & & Presentación & $\begin{array}{l}\text { ¿Contó con presentación } \\
\text { individual y con todos los } \\
\text { elementos para trabajar } \\
\text { en el campo? }\end{array}$ & Sí (1), no (0) & 5 & $(0-5)$ \\
\hline & & Autorización & $\begin{array}{l}\text { ¿Contó con autorización } \\
\text { del Invima? }\end{array}$ & Sí (1), no (0) & 5 & $(0-5)$ \\
\hline & & $\begin{array}{l}\text { Conocimiento de la prueba } \\
\text { en la región }\end{array}$ & $\begin{array}{l}\text { ¿Existen estudios y } \\
\text { publicaciones en Colombia } \\
\text { sobre la sensibilidad y } \\
\text { especificidad de la PDR? }\end{array}$ & Sí (1), no (0) & 4 & $(0-4)$ \\
\hline
\end{tabular}

Total tema A partir del producto del nivel de satisfacción y la ponderación para cada indicador, se calcula el porcentaje de cumplimiento dividiendo el valor obtenido por el valor máximo del intervalo de la variable y multiplicándolo por 100. El porcentaje de cumplimiento corresponde al mismo de la variable. Se promedia el cumplimiento de cada variable para determinar el del tema general. Se estimó como cumplimiento adecuado un porcentaje $\geq 70 \%$.

componente

Garantía

Implementación Control de calidad

de la calidad

con paneles

Distribución de las PDR

de acuerdo con las

necesidades

Capacidad técnica de los trabajadores de la salud y colaboradores

comunitarios en los diez departamentos

Evaluación del desempeño de los trabajadores de la salud
¿Contó con controles de calidad realizados con paneles?

Hubo distribución de acuerdo con las necesidades en cada uno de los 10 departamentos?

¿Se capacitaron en el tema trabajadores de la salud y colaboradores comunitarios en los diez departamentos

¿Se cumplió con las directrices para la evaluación del desempeño de los trabajadores de salud que realizaron el diagnóstico por PDR?
Si (1), no (0)

4

Nulo (0), parcial (1), total (2)

5

Sí (1), no (0)

5

Nulo (0), parcial (1) total (2)

5 


\begin{tabular}{|c|c|c|c|c|c|c|}
\hline Componente & Tema & Variable & Indicador & $\begin{array}{l}\text { Niveles de } \\
\text { satisfacción }\end{array}$ & Ponderación & $\begin{array}{c}\text { Intervalo } \\
\text { de la variable }\end{array}$ \\
\hline & & $\begin{array}{l}\text { Distribución de indicaciones } \\
\text { escritas para el uso de las } \\
\text { PDR por parte de los } \\
\text { departamentos }\end{array}$ & $\begin{array}{l}\text { ¿Se distribuyeron } \\
\text { directrices escritas } \\
\text { para el uso adecuado } \\
\text { de PDR? }\end{array}$ & Sí (1), no (0) & 5 & $(0-5)$ \\
\hline & & $\begin{array}{l}\text { Cumplimiento de directrices } \\
\text { de uso de las PDR } \\
\text { (población objeto) }\end{array}$ & $\begin{array}{l}\text { ¿Se cumplieron las directrices } \\
\text { de aplicación por parte } \\
\text { de los departamentos con } \\
\text { respecto a la población } \\
\text { objeto? }\end{array}$ & $\begin{array}{l}\text { Nulo (0), parcial (1), } \\
\text { total (2) }\end{array}$ & 4 & $(0-8)$ \\
\hline & & $\begin{array}{l}\text { Encuesta de percepción } \\
\text { de los trabajadores de } \\
\text { salud frente a las PDR }\end{array}$ & $\begin{array}{l}\text { ¿Se realizó encuesta } \\
\text { por departamento } \\
\text { para determinar la } \\
\text { percepción de los } \\
\text { trabajadores de salud } \\
\text { frente a las PDR? }\end{array}$ & Sí (1), no (0) & 3 & $(0-3)$ \\
\hline & & $\begin{array}{l}\text { Encuesta de percepción } \\
\text { de la comunidad frente a } \\
\text { las PDR }\end{array}$ & $\begin{array}{l}\text { ¿Se realizó encuesta } \\
\text { por departamento } \\
\text { para determinar la } \\
\text { percepción de la } \\
\text { comunidad frente a } \\
\text { las PDR? }\end{array}$ & $\mathrm{Si}(1)$, no $(0)$ & 4 & $(0-4)$ \\
\hline & & $\begin{array}{l}\text { Cumplimiento de } \\
\text { directrices sobre } \\
\text { temperatura y humedad de } \\
\text { las PDR }\end{array}$ & $\begin{array}{l}\text { ¿Hubo cumplimiento } \\
\text { de directrices } \\
\text { sobre temperatura } \\
\text { y humedad en el } \\
\text { transporte y } \\
\text { almacenamiento de } \\
\text { las PDR, nivel central- } \\
\text { Pamafro (Perú)? }\end{array}$ & $\begin{array}{l}\text { Nulo (0), parcial (1), } \\
\text { total (2) }\end{array}$ & 5 & $(0-10)$ \\
\hline & & & $\begin{array}{l}\text { ¿Hubo cumplimiento } \\
\text { de directrices sobre } \\
\text { temperatura y humedad } \\
\text { en el transporte y } \\
\text { almacenamiento de las } \\
\text { PDR, nivel nacional- } \\
\text { Pamafro (Colombia)? }\end{array}$ & $\begin{array}{l}\text { Nulo (0), parcial (1), } \\
\text { total (2) }\end{array}$ & 5 & $(0-10)$ \\
\hline & & & $\begin{array}{l}\text { ¿Hubo cumplimiento } \\
\text { de directrices sobre } \\
\text { temperatura y humedad } \\
\text { en el transporte, } \\
\text { almacenamiento y } \\
\text { lugar de uso de las PDR, } \\
\text { nivel departamental? }\end{array}$ & $\begin{array}{l}\text { Nulo (1), parcial (2), } \\
\text { total (3) }\end{array}$ & 5 & $(0-10)$ \\
\hline & & Logística & $\begin{array}{l}\text { ¿Se contó con la logística } \\
\text { (transporte) adecuada } \\
\text { para la puesta en marcha } \\
\text { de la estrategia hacia el } \\
\text { nivel nacional? }\end{array}$ & $\begin{array}{l}\text { Nulo }(0) \text {, parcial (1), } \\
\text { total (2) }\end{array}$ & 4 & $(0-8)$ \\
\hline & & & $\begin{array}{l}\text { ¿Se contó con la logística } \\
\text { (transporte) adecuada } \\
\text { para la puesta en marcha } \\
\text { de la estrategia hacia el } \\
\text { nivel departamental? }\end{array}$ & $\begin{array}{l}\text { Nulo (0), parcial (1), } \\
\text { total (2) }\end{array}$ & 4 & $(0-8)$ \\
\hline & & & $\begin{array}{l}\text { ¿Se contó con la logística } \\
\text { (transporte) adecuada } \\
\text { para la puesta en marcha } \\
\text { de la estrategia del nivel } \\
\text { departamental al } \\
\text { municipal? }\end{array}$ & $\begin{array}{l}\text { Nulo (0), parcial (1), } \\
\text { total (2) }\end{array}$ & 5 & $(0-10)$ \\
\hline & & Notificación & $\begin{array}{l}\text { ¿El nivel departamental } \\
\text { reportó los casos a } \\
\text { SIVIGILA? }\end{array}$ & Si (1), no (0). & 5 & $(0-5)$ \\
\hline & & Supervisión & $\begin{array}{l}\text { ¿Se contó con } \\
\text { supervisiones en campo } \\
\text { realizadas por el nivel } \\
\text { nacional-Pamafro a los } \\
\text { departamentos? }\end{array}$ & $\begin{array}{l}\text { Nulo }(0) \text {, parcial (1), } \\
\text { total (2) }\end{array}$ & 4 & $(0-8)$ \\
\hline & & & $\begin{array}{l}\text { ¿Se contó con } \\
\text { supervisiones en campo } \\
\text { realizadas por el nivel } \\
\text { departamental a las } \\
\text { localidades? }\end{array}$ & $\begin{array}{l}\text { Nulo }(0) \text {, parcial (1), } \\
\text { total (2) }\end{array}$ & 5 & $(0-10)$ \\
\hline
\end{tabular}




\begin{tabular}{|c|c|c|c|c|c|c|}
\hline Componente & Tema & Variable & Indicador & $\begin{array}{l}\text { Niveles de } \\
\text { satisfacción }\end{array}$ & Ponderación & $\begin{array}{c}\text { Intervalo } \\
\text { de la variable }\end{array}$ \\
\hline & Evaluación & $\begin{array}{l}\text { Evaluación de resultado, } \\
\text { uso y cobertura de las } \\
\text { PDR }\end{array}$ & $\begin{array}{l}\text { ¿Se realizó evaluación } \\
\text { de resultado, uso } \\
\text { y cobertura? }\end{array}$ & Sí (1), no (0) & 4 & $(0-4)$ \\
\hline \multirow[t]{16}{*}{$\begin{array}{l}\text { Garantía } \\
\text { de la calidad }\end{array}$} & Implementación & $\begin{array}{l}\text { Control de calidad } \\
\text { con paneles }\end{array}$ & $\begin{array}{l}\text { ¿Contó con controles } \\
\text { de calidad realizados } \\
\text { con paneles? }\end{array}$ & Sí (1), no (0) & 4 & $(0-4)$ \\
\hline & & $\begin{array}{l}\text { Distribución de las PDR } \\
\text { de acuerdo con las } \\
\text { necesidades }\end{array}$ & $\begin{array}{l}\text { ¿Hubo distribución de } \\
\text { acuerdo con las necesidades } \\
\text { en cada uno de los } 10 \\
\text { departamentos? }\end{array}$ & $\begin{array}{l}\text { Nulo }(0) \text {, parcial }(1) \text {, } \\
\text { total }(2)\end{array}$ & 5 & $(0-10)$ \\
\hline & & $\begin{array}{l}\text { Capacidad técnica de los } \\
\text { trabajadores de la salud y } \\
\text { colaboradores comunitarios en } \\
\text { los diez departamentos }\end{array}$ & $\begin{array}{l}\text { ¿Se capacitó personal en } \\
\text { el tema entre trabajadores } \\
\text { de la salud y colaboradores } \\
\text { comunitarios en lo diez } \\
\text { departamentos? }\end{array}$ & Sí (1), no (0) & 5 & $(0-5)$ \\
\hline & & $\begin{array}{l}\text { Evaluación del desempeño de } \\
\text { los trabajadores de la salud }\end{array}$ & $\begin{array}{l}\text { ¿Se cumplió con las directrices } \\
\text { para la evaluación del } \\
\text { desempeño a los trabajadores } \\
\text { de salud que realizaron el } \\
\text { diagnóstico por PDR? }\end{array}$ & $\begin{array}{l}\text { Nulo }(0) \text {, parcial }(1) \text {, } \\
\text { total }(2)\end{array}$ & 5 & $(0-10)$ \\
\hline & & $\begin{array}{l}\text { Distribución de indicaciones } \\
\text { escritas para el uso de las PDR } \\
\text { por parte de los departamentos }\end{array}$ & $\begin{array}{l}\text { ¿Se distribuyeron directrices } \\
\text { escritas para el uso adecuado } \\
\text { de las PDR? }\end{array}$ & Sí (1), no (0) & 5 & $(0-5)$ \\
\hline & & $\begin{array}{l}\text { Cumplimiento de directrices } \\
\text { de uso de PDR (población objeto) }\end{array}$ & $\begin{array}{l}\text { ¿Se cumplieron las directrices } \\
\text { de aplicación por parte de los } \\
\text { departamentos con respecto } \\
\text { a la población objeto? }\end{array}$ & $\begin{array}{l}\text { Nulo }(0) \text {, parcial }(1) \text {, } \\
\text { total }(2)\end{array}$ & 4 & $(0-8)$ \\
\hline & & $\begin{array}{l}\text { Encuesta de percepción de } \\
\text { los trabajadores de salud frente } \\
\text { a las PDR }\end{array}$ & $\begin{array}{l}\text { ¿Se realizó encuesta por } \\
\text { departamento para determinar } \\
\text { la percepción de los } \\
\text { trabajadores de salud frente } \\
\text { a las PDR? }\end{array}$ & Sí (1), no (0) & 3 & $(0-3)$ \\
\hline & & $\begin{array}{l}\text { Encuesta de percepción de la } \\
\text { comunidad frente a las PDR }\end{array}$ & $\begin{array}{l}\text { ¿Se realizó encuesta por } \\
\text { departamento para determinar } \\
\text { la percepción de la comunidad } \\
\text { frente a las PDR? }\end{array}$ & Sí (1), no (0) & 4 & $(0-4)$ \\
\hline & & $\begin{array}{l}\text { Cumplimiento de directrices } \\
\text { temperatura y humedad de } \\
\text { las PDR }\end{array}$ & $\begin{array}{l}\text { ¿Hubo cumplimiento de } \\
\text { directrices sobre temperatura } \\
\text { y humedad en el transporte } \\
\text { y almacenamiento de las PDR, } \\
\text { nivel central-Pamafro (Perú)? }\end{array}$ & $\begin{array}{l}\text { Nulo }(0) \text {, parcial }(1) \text {, } \\
\text { total }(2)\end{array}$ & 5 & $(0-10)$ \\
\hline & & & $\begin{array}{l}\text { ¿Hubo cumplimiento de } \\
\text { directrices sobre temperatura }\end{array}$ & $\begin{array}{l}\text { Nulo }(0) \text {, parcial }(1) \text {, } \\
\text { total (2) }\end{array}$ & 5 & $(0-10)$ \\
\hline & & & $\begin{array}{l}\text { y humedad en el transporte } \\
\text { y almacenamiento de las } \\
\text { PDR, nivel nacional-Pamafro } \\
\text { (Colombia)? }\end{array}$ & & & \\
\hline & & & $\begin{array}{l}\text { ¿Hubo cumplimiento de } \\
\text { directrices sobre } \\
\text { temperatura y humedad } \\
\text { en el transporte, } \\
\text { almacenamiento y lugar de } \\
\text { uso de las PDR, nivel } \\
\text { departamental? }\end{array}$ & $\begin{array}{l}\text { Nulo (1), parcial (2), } \\
\text { total (3) }\end{array}$ & 5 & $(0-10)$ \\
\hline & & Logística & $\begin{array}{l}\text { ¿Se contó con la logística } \\
\text { (transporte) adecuada para } \\
\text { la puesta en marcha de la } \\
\text { estrategia hacia el nivel } \\
\text { nacional? }\end{array}$ & $\begin{array}{l}\text { Nulo }(0) \text {, parcial (1), } \\
\text { total (2) }\end{array}$ & 4 & $(0-8)$ \\
\hline & & & $\begin{array}{l}\text { ¿Se contó con la logística } \\
\text { (transporte) adecuada } \\
\text { para la puesta en marcha } \\
\text { de la estrategia hacia el } \\
\text { nivel departamental? }\end{array}$ & $\begin{array}{l}\text { Nulo }(0) \text {, parcial }(1) \text {, } \\
\text { total }(2)\end{array}$ & 4 & $(0-8)$ \\
\hline & & & $\begin{array}{l}\text { ¿Se contó con la logística } \\
\text { (transporte) adecuada para } \\
\text { la puesta en marcha de la } \\
\text { estrategia del nivel } \\
\text { departamental al municipal? }\end{array}$ & $\begin{array}{l}\text { Nulo }(0) \text {, parcial }(1) \text {, } \\
\text { total }(2)\end{array}$ & 5 & $(0-10)$ \\
\hline & & Notificación & $\begin{array}{l}\text { ¿El nivel departamental reportó } \\
\text { los casos a SIVIGILA? }\end{array}$ & Sí (1), no (0) & 5 & $(0-5)$ \\
\hline
\end{tabular}




\begin{tabular}{|c|c|c|c|c|c|c|}
\hline Componente & Tema & Variable & Indicador & $\begin{array}{l}\text { Niveles de } \\
\text { satisfacción }\end{array}$ & Ponderación & $\begin{array}{c}\text { Intervalo } \\
\text { de la variable }\end{array}$ \\
\hline & & Supervisión & $\begin{array}{l}\text { ¿Se contó con supervisiones } \\
\text { en campo realizadas por el } \\
\text { nivel nacional-Pamafro } \\
\text { a los departamentos? }\end{array}$ & $\begin{array}{l}\text { Nulo (0), parcial (1), } \\
\text { total (2) }\end{array}$ & 4 & $(0-12)$ \\
\hline & & & $\begin{array}{l}\text { ¿Se contó con supervisiones } \\
\text { en campo realizadas por el } \\
\text { nivel departamental } \\
\text { a las localidades? }\end{array}$ & $\begin{array}{l}\text { Nulo }(0) \text {, parcial (1), } \\
\text { total (2) }\end{array}$ & 5 & $(0-10)$ \\
\hline & Evaluación & $\begin{array}{l}\text { Evaluación de } \\
\text { resultado, } \\
\text { uso y cobertura } \\
\text { de las PDR }\end{array}$ & $\begin{array}{l}\text { ¿Se realizó evaluación de } \\
\text { resultado, uso y cobertura? }\end{array}$ & Sí (1) no (0) & 4 & $(0-4)$ \\
\hline Total tema & \multicolumn{6}{|c|}{$\begin{array}{l}\text { A partir del producto del nivel de satisfacción y la ponderación para cada indicador, se calcula el porcentaje de cumplimiento dividiendo el } \\
\text { valor obtenido por el valor máximo del intervalo de la variable y se multiplica por } 100 \text {. Cuando existe más de un indicador por variable se } \\
\text { deben promediar los resultados para determinar el porcentaje de cumplimiento de la variable. Se promedia el cumplimiento de cada variable } \\
\text { para determinar el del tema general. Se estimó como cumplimiento adecuado un porcentaje } \geq 70 \% \text {. }\end{array}$} \\
\hline $\begin{array}{l}\text { Total } \\
\text { componente }\end{array}$ & \multicolumn{6}{|c|}{ El porcentaje de cumplimiento para este componente corresponde al promedio de los dos temas. } \\
\hline
\end{tabular}

PDR: pruebas de diagnóstico rápido

Definición del indicador: expresa el porcentaje de epidemias o brotes que fueron intervenidos con pruebas de diagnóstico rápido en los departamentos objeto, como estrategia integral del programa.

Nombre del indicador. Número de búsquedas activas con aplicación de pruebas de diagnóstico rápido en Colombia durante la intervención.

Definición del indicador: expresa el número de búsquedas activas realizadas en los departamentos objeto como parte de una estrategia integral del programa de malaria.

- Indicador de uso

Nombre del indicador. Porcentaje de pruebas de diagnóstico rápido utilizadas en Colombia durante el período intervenido.

Definición del indicador: expresa la proporción de pruebas de diagnóstico rápido utilizadas del total de las distribuidas en los departamentos intervenidos.

- Indicador de cobertura

Nombre del indicador. Porcentaje de localidades objeto del proyecto que recibieron pruebas de diagnóstico rápido en Colombia.

Definición del indicador: expresa la disponibilidad de pruebas de diagnóstico rápido para malaria en las localidades objeto del proyecto.

Toda la información de la aplicación de pruebas de diagnóstico rápido en Colombia fue sistematizada en bases de datos en (Microsoft Excel 2007 (B).

\section{Resultados}

Evaluación del cumplimiento del ciclo de implementación del uso de las pruebas de diagnóstico rápido

El cumplimiento general del ciclo de implementación del uso de las pruebas de diagnóstico rápido fue de $71 \%$, el cual resultó del promedio de cumplimiento de los componentes: planeación (50\%), adquisición (83 \%) y garantía de la calidad (80\%). El cuadro 2 presenta los porcentajes de cumplimiento por indicador, variable y tema en cada uno de estos componentes.

\section{Evaluación de los indicadores de resultado, uso y cobertura de la estrategia de implementación de las pruebas de diagnóstico rápido}

Indicadores de resultado

Casos de malaria determinados mediante pruebas de diagnóstico rápido en Colombia: se encontró que, en los cinco años anteriores a la aplicación de pruebas de diagnóstico rápido, ninguno de los departamentos utilizó este método para diagnosticar malaria. Sin embargo, de los 11.169 casos reportados por los departamentos desde octubre de 2007 a julio de 2008, el 17,4 \% se detectó con pruebas de diagnóstico rápido y el porcentaje restante, con gota gruesa.

El cuadro 3 muestra el número de diagnósticos realizados y casos detectados por pruebas de diagnóstico rápido, por departamento intervenido.

Porcentaje de pacientes con malaria detectados con pruebas de diagnóstico rápido y que recibieron 
Cuadro 2. Porcentajes de cumplimiento por indicador, variable y tema en cada uno los componentes del ciclo de implementación.

\begin{tabular}{|c|c|c|c|c|}
\hline Componente & Tema & Variable & Indicador & $\begin{array}{l}\text { Puntuación } \\
\text { variable } \\
\text { crítica }\end{array}$ \\
\hline \multirow[t]{4}{*}{ Planeación (50\%) } & $\begin{array}{l}\text { Identificación de áreas } \\
(50 \%)\end{array}$ & $\begin{array}{l}\text { Estudio de necesidades } \\
\text { en el país }(50 \%)\end{array}$ & $\begin{array}{l}\text { ¿Hubo consulta adecuada } \\
\text { del nivel central sobre las } \\
\text { necesidades de PDR para } \\
\text { Colombia? }(50 \%)\end{array}$ & 5 \\
\hline & & $\begin{array}{l}\text { Estudio de necesidades } \\
\text { en los departamentos } \\
(50 \%)\end{array}$ & $\begin{array}{l}\text { ¿Hubo consulta adecuada } \\
\text { de las necesidades de } \\
\text { PDR en el nivel } \\
\text { departamental? (50 \%) }\end{array}$ & 5 \\
\hline & & $\begin{array}{l}\text { Estudios de costo- } \\
\text { efectividad para la } \\
\text { aplicación de las } \\
\text { PDR }(0 \%)\end{array}$ & $\begin{array}{l}\text { ¿Existieron estudios de costo- } \\
\text { efectividad para decidir } \\
\text { la aplicación de las PDR } \\
\text { en el país? }(0 \%)\end{array}$ & - \\
\hline & & $\begin{array}{l}\text { Diseño de directrices } \\
\text { a los departamentos } \\
(100 \%)\end{array}$ & $\begin{array}{l}\text { ¿Se diseñaron directrices } \\
\text { escritas para la selección } \\
\text { del área de aplicación o } \\
\text { situación en la que debía } \\
\text { aplicarse la PDR por } \\
\text { departamento? (100 \%) }\end{array}$ & 5 \\
\hline \multirow[t]{6}{*}{ Adquisición (83\%) } & $\begin{array}{l}\text { Selección de la PDR } \\
(83 \%)\end{array}$ & $\begin{array}{l}\text { Capacidad para diferenciar } \\
\text { infección por } P \text {. falciparum } \\
\text { de otras especies (antígenos } \\
\text { blanco) }(100 \%)\end{array}$ & $\begin{array}{l}\text { ¿La prueba contó con } \\
\text { antígeno panpalúdico y } \\
\text { antígeno específico para } \\
P \text {. falciparum? (100 \%) }\end{array}$ & 5 \\
\hline & & Costo de la prueba (0 \%) & $\begin{array}{l}\text { ¿El costo fue inferior a } \\
\text { US\$ } 1,5 ?(0 \%)\end{array}$ & 0 \\
\hline & & $\begin{array}{l}\text { Capacidad para realizar } \\
\text { controles al tratamiento } \\
(100 \%)\end{array}$ & $\begin{array}{l}\text { ¿El antígeno decrece } \\
\text { proporcionalmente con } \\
\text { la parasitemia? (100\%) }\end{array}$ & 3 \\
\hline & & Presentación (100%) & $\begin{array}{l}\text { ¿Contó con presentación } \\
\text { individual y con todos los } \\
\text { elementos para trabajar } \\
\text { en el campo? }(100 \%)\end{array}$ & 5 \\
\hline & & Autorización (100%) & $\begin{array}{l}\text { ¿Contó con autorización } \\
\text { del INVIMA? (100\%) }\end{array}$ & 5 \\
\hline & & $\begin{array}{l}\text { Conocimiento de la prueba } \\
\text { en la región (100 \%) }\end{array}$ & $\begin{array}{l}\text { ¿Existen estudios y } \\
\text { publicaciones en Colombia } \\
\text { sobre la sensibilidad y } \\
\text { especificidad de la PDR? } \\
(100 \%)\end{array}$ & 4 \\
\hline \multirow[t]{5}{*}{$\begin{array}{l}\text { Garantía de la } \\
\text { calidad (80\%) }\end{array}$} & Implementación (60%) & $\begin{array}{l}\text { Control de calidad } \\
\text { con paneles }(0 \%)\end{array}$ & $\begin{array}{l}\text { ¿Contó con controles } \\
\text { de calidad realizados } \\
\text { con paneles? }(0 \%)\end{array}$ & 0 \\
\hline & & $\begin{array}{l}\text { Distribución de las PDR } \\
\text { de acuerdo con las } \\
\text { necesidades (50 \%) }\end{array}$ & $\begin{array}{l}\text { ¿Hubo distribución de } \\
\text { acuerdo con las necesidades } \\
\text { en cada uno de los } 10 \\
\text { departamentos? }(50 \%)\end{array}$ & 5 \\
\hline & & $\begin{array}{l}\text { Capacidad técnica de los } \\
\text { trabajadores de la salud y } \\
\text { colaboradores comunitarios } \\
\text { en los diez departamentos } \\
(100 \%)\end{array}$ & $\begin{array}{l}\text { ¿Se capacitó personal } \\
\text { en el tema entre } \\
\text { trabajadores de la salud } \\
\text { y colaboradores comunitarios } \\
\text { en lo diez departamentos? } \\
(100 \%)\end{array}$ & 5 \\
\hline & & $\begin{array}{l}\text { Evaluación del desempeño } \\
\text { de los trabajadores de la } \\
\text { salud }(50 \%)\end{array}$ & $\begin{array}{l}\text { ¿Se cumplió con las } \\
\text { directrices para la evaluación } \\
\text { del desempeño a los } \\
\text { trabajadores de salud que } \\
\text { realizaron el diagnóstico por } \\
\text { PDR? }(50 \%)\end{array}$ & 5 \\
\hline & & $\begin{array}{l}\text { Distribución de indicaciones } \\
\text { escritas para el uso de las } \\
\text { PDR por parte de los } \\
\text { departamentos (100 \%) }\end{array}$ & $\begin{array}{l}\text { ¿Se distribuyeron directrices } \\
\text { escritas para el uso } \\
\text { adecuado de las PDR? } \\
(100 \%)\end{array}$ & 5 \\
\hline
\end{tabular}




\begin{tabular}{|c|c|c|c|}
\hline Componente & Tema & Variable & Indicador \\
\hline
\end{tabular}

Cumplimiento de directrices de uso de las PDR (población objeto) (50 \%)

Encuesta de percepción de los trabajadores de salud frente a las PDR (100\%)

Encuesta de percepción de la comunidad frente a las PDR (0\%)

Cumplimiento de directrices temperatura y humedad de las PDR (83\%)

Logística (67\%)

Notificación (100\%)

Supervisión (25\%)
¿Se cumplieron las directrices de aplicación por parte de los departamentos con respecto a la población objeto? (50\%)

¿Se realizó encuesta por departamento para determinar la percepción de los trabajadores de salud frente a las PDR? (100\%)

¿Se realizó encuesta por departamento para determinar la percepción de la comunidad frente a las PDR? (0 \%)

¿Hubo cumplimiento de directrices sobre temperatura y humedad en el transporte y almacenamiento de PDR, nivel central-Pamafro

(Perú)? (100\%)

¿Hubo cumplimiento de directrices sobre temperatura y humedad en el transporte y almacenamiento de PDR, nivel nacional-Pamafro (Colombia)? (100\%)

¿Hubo cumplimiento de directrices sobre temperatura y humedad en el transporte, almacenamiento y lugar de uso de las PDR, nivel departamental? (50\%)

¿Se contó con la logística (transporte) adecuada para la puesta en marcha de la estrategia hacia el nivel nacional? (100\%)

¿Se contó con la logística (transporte) adecuada para la puesta en marcha de la estrategia hacia el nivel departamental? (50\%)

¿Se contó con la logística (transporte) adecuada para la puesta en marcha de la estrategia del nivel departamental al municipal? (50\%)

¿El nivel departamental reportó los casos a SIVIGILA? (100\%)

¿Se contó con supervisiones en campo realizada por el nivel nacional-Pamafro a los departamentos? $(0 \%)$

¿Se contó con supervisiones en campo realizada por el nivel departamental a las localidades? $(50 \%)$

Evaluación de resultado, uso y ¿Se realizó evaluación de cobertura de las PDR (100) resultado, uso y cobertura de las PDR? (100 \%)

4 


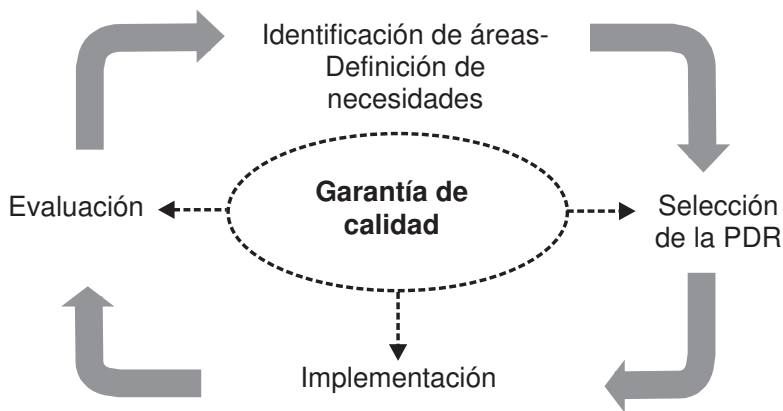

Figura 1. Ciclo de implementación del uso de las pruebas de diagnóstico rápido

PDR: prueba de diagnóstico rápido

tratamiento antipalúdico: fueron tratados 1.939 pacientes $(99,9 \%)$ de los 1.940 con diagnóstico positivo mediante estas pruebas. El departamento de Guainía hizo diagnóstico mediante gota gruesa y pruebas de diagnóstico rápido simultáneamente en los pacientes atendidos, por lo que uno de ellos no recibió tratamiento por ser negativo por microscopía.

Porcentaje de epidemias o brotes intervenidos con pruebas de diagnóstico rápido en Colombia durante la intervención: el porcentaje de intervención obtenido fue del $100 \%$, debido a que el único brote epidémico reportado durante el período de aplicación de las pruebas se registró en La Guajira, desde la semana epidemiológica 50 de 2007 hasta la semana epidemiológica 08 de 2008, en el que las medidas de intervención aplicadas incluyeron las pruebas de diagnóstico rápido (11).

El brote fue atendido realizando búsqueda activa casa a casa y el diagnóstico por estas pruebas se aplicó a toda la población febril y a los contactos. Se realizó gota gruesa al $100 \%$ de los pacientes detectados por pruebas de diagnóstico rápido; las gotas gruesas fueron leídas en el $100 \%$ en la actividad de control de calidad.

Los pacientes positivos se trataron basándose en las pruebas de diagnóstico rápido y siguiendo los lineamientos del tratamiento de acuerdo con la normatividad vigente del Ministerio de la Protección Social.

Número de búsquedas activas con aplicación de pruebas de diagnóstico rápido en Colombia durante la intervención: se llevaron a cabo 218 jornadas de búsqueda activa como intervención de prevención y control en los departamentos objeto del proyecto. La participación de los departamentos en esta actividad se aprecia en la figura 2.

En la presente evaluación se encontró que la búsqueda activa se hizo en las siguientes circunstancias:

1. En localidades palúdicas distantes se hizo el "barrido hemático" a toda la población, tanto febril como no febril. El barrido hemático consistió en hacer el diagnóstico de malaria tanto con pruebas de diagnóstico rápido como por gota gruesa a toda la población en riesgo. En localidades cercanas se aplicaron estas pruebas a los pacientes febriles y a sus contactos.

2. Se aplicó a la población indígena flotante.

3. En las localidades palúdicas, las pruebas de diagnóstico rápido se practicaron a todo paciente febril. Estas localidades solamente cumplían con el requisito de tener transmisión de malaria, independientemente de su ubicación; el punto de interés fue enfocar la búsqueda activa en la población febril. También se practicaron como control de las personas que ingresaban por el puerto del municipio.

Cuadro 3. Número de diagnósticos realizados y casos detectados mediante pruebas de diagnóstico rápido en los departamentos objeto durante el período de aplicación.

\begin{tabular}{|c|c|c|c|}
\hline Departamento & $\begin{array}{c}\text { Número de pruebas } \\
\text { aplicadas }\end{array}$ & $\begin{array}{c}\text { Número de pruebas } \\
\text { positivas }\end{array}$ & $\begin{array}{c}\text { Porcentaje de pruebas } \\
\text { positivas (\%) }\end{array}$ \\
\hline Amazonas & 1.277 & 114 & 9 \\
\hline Arauca & 1.250 & 16 & 1 \\
\hline Boyacá & 168 & 0 & 0 \\
\hline Cesar & 21 & 0 & 0 \\
\hline Guainía & 452 & 50 & 11 \\
\hline La Guajira & 2.237 & 729 & 33 \\
\hline Nariño & 1.263 & 385 & 30 \\
\hline Norte de Santander & 523 & 60 & 11 \\
\hline Vichada & 478 & 29 & 6 \\
\hline Putumayo & 13.931 & 557 & 4 \\
\hline Total & 21.600 & 1.940 & 9 \\
\hline
\end{tabular}


4. En situación de epidemia, se hizo mediante búsqueda activa casa a casa dirigida a los pacientes febriles y a sus contactos.

\section{Indicador de uso}

Porcentaje de pruebas de diagnóstico rápido utilizadas en Colombia durante el período intervenido: el cuadro 4 muestra el total de pruebas de diagnóstico rápido distribuidas, frente al total de muestras utilizadas en el diagnóstico de malaria por departamento y las pruebas faltantes, entendiéndose por faltantes aquellas que no se utilizaron en el diagnóstico de pacientes sino que se vencieron o se utilizaron en actividades de capacitación en el departamento o cuyos resultados de aplicación se desconocen o que fueron objeto de decomiso en campo.

De las 58.008 pruebas entregadas a Colombia, se distribuyeron a los departamentos objeto 31.281 pruebas (54\%), de las cuales, se utilizó $69, \%$ en el diagnóstico de malaria. Los departamentos que tuvieron un mayor porcentaje de pruebas utilizadas fueron Boyacá, Cesar y Arauca. El $46 \%$ de las pruebas restantes entregadas a Colombia se distribuyó a los departamentos de Valle del Cauca, Córdoba y Chocó, que manifestaron la necesidad de hacer uso de las pruebas rápidas debido a la problemática en salud pública que representa la malaria en estos sitios. De igual manera, se entregaron pruebas al Instituto Nacional de Salud, a la Agencia Presidencial para la Acción Social y a PAMAFRO Colombia, para capacitación y entrenamiento en pruebas de diagnóstico rápido.

Se determinó que $0,2 \%$ de las pruebas fueron utilizadas para repeticiones de los diagnósticos, en los casos que se presentó duda por parte del personal encargado de esta actividad.

\section{Indicador de cobertura}

Porcentaje de localidades objeto del proyecto que recibieron pruebas de diagnóstico rápido en Colombia: en los diez departamentos, el total

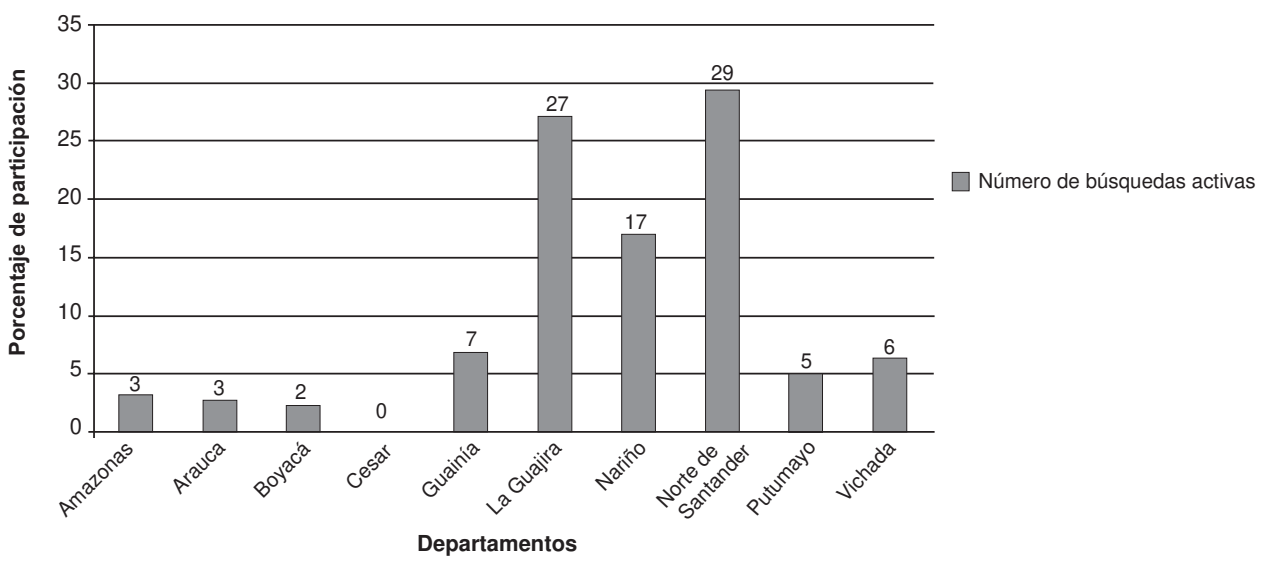

Figura 2. Participación de los departamentos en la actividad de búsqueda activa realizada con pruebas de diagnóstico rápido.

Cuadro 4. Relación de las pruebas de diagnóstico rápido utilizadas por departamento desde octubre de 2007 hasta julio de 2008.

\begin{tabular}{lcccr}
\hline Departamento & $\begin{array}{c}\text { Pruebas } \\
\text { distribuidas }\end{array}$ & $\begin{array}{c}\text { Pruebas } \\
\text { utilizadas }\end{array}$ & $\begin{array}{c}\text { Porcentaje de } \\
\text { pruebas utilizadas }\end{array}$ & $\begin{array}{c}\text { Pruebas } \\
\text { faltantes }\end{array}$ \\
\hline Amazonas & 1.692 & 1.277 & 75 & 415 \\
Arauca & 1.484 & 1.250 & 84 & 234 \\
Boyacá & 168 & 168 & 100 & 0 \\
Cesar & 24 & 21 & 88 & 3 \\
Guainía & 1.128 & 452 & 40 & 676 \\
La Guajira & 3.504 & 2.237 & 64 & 1.267 \\
Nariño & 2.904 & 1.263 & 43 & 1.641 \\
Norte de Santander & 792 & 523 & 66 & 269 \\
Putumayo & 16.684 & 13.931 & 83 & 2.753 \\
Vichada & 2904 & 478 & 69 & 2.426 \\
Total & 31.284 & 21.600 & 69 & 9.684 \\
\hline
\end{tabular}


de localidades por intervenir por el proyecto PAMAFRO para realizar actividades de prevención y control fueron 3.374, de las cuales, tuvieron cobertura con pruebas de diagnóstico rápido el $14 \%$ (472). Sin embargo, teniendo en cuenta el criterio técnico en cada departamento, se aumentó la cobertura a otras localidades con paludismo no programadas. Por lo tanto, hubo disponibilidad de pruebas de diagnóstico rápido en $25 \%$ (861) de estas localidades.

\section{Discusión}

Los resultados de la evaluación de la aplicación de las pruebas de diagnóstico rápido en Colombia dentro del proyecto PAMAFRO, pueden considerarse para estructurar lineamientos y políticas del orden nacional para el control de la malaria.

En lo que respecta al cumplimiento del ciclo de implementación de las pruebas, el componente más deficiente fue la planeación, específicamente en el tema de la identificación del área de aplicación.

Consideramos necesario resaltar y profundizar aquellas variables que presentaron porcentajes de cumplimiento inferior al $70 \%$ y que, además, fueron clasificadas como "variables críticas", por encontrar en ellas una oportunidad de mejora (cuadro 2).

En el componente de planeación, es importante fortalecer las variables "estudio de las necesidades de pruebas de diagnóstico rápido en el país" y "estudio de necesidades en los departamentos", las cuales tienen relación directa con la selección del área de aplicación y con la cantidad de pruebas que deben distribuirse por departamentos.

La planeación tiene gran importancia en las estrategias de implementación debido a que determina el horizonte, los protocolos, las circunstancias, las actividades, los tiempos y las cantidades para armonizar los lineamientos nacionales con las necesidades locales. Por otra parte, una planeación adecuada previene situaciones futuras, pretendiendo reducir riesgos y aprovechando las oportunidades para un adecuado desarrollo de las actividades propuestas en el plan de trabajo.

Dentro de la planeación de las adquisiciones y teniendo presente que en ocasiones existen problemas de tipo administrativo y logístico, es adecuado garantizar que las pruebas de diagnóstico rápido adquiridas cuenten con una vida útil con un mínimo de 18 meses de vigencia una vez realizada la compra (7) y se garantice el transporte adecuado hacia los lugares de aplicación.

Con el fin de estimar apropiadamente las necesidades de las pruebas de diagnóstico rápido y evitar eventuales pérdidas de recursos, es recomendable hacer un diagnóstico de las necesidades locales de las pruebas, para lo cual se puede contar con una justificación técnica ajustada a las necesidades de la zona que se va a intervenir, la cual debe ser elaborada por el personal responsable del programa del lugar, en coordinación con su grupo técnico de trabajo.

Dentro del componente de garantía de calidad, las variables de especial interés son "distribución de las pruebas de acuerdo con las necesidades", "evaluación del desempeño de los trabajadores de salud", "cumplimiento de directrices sobre temperatura y humedad en el nivel departamental", "logística" y "supervisión".

Cuando se busca fortalecer la red de diagnóstico de malaria con un diagnóstico complementario como las pruebas de diagnóstico rápido, es necesario posibilitar el uso adecuado de las pruebas en campo y que exista coherencia entre

Cuadro 4. Relación de las pruebas de diagnóstico rápido utilizadas por departamento desde octubre de 2007 hasta julio de 2008.

\begin{tabular}{lcccr}
\hline Departamento & $\begin{array}{c}\text { Pruebas } \\
\text { distribuidas }\end{array}$ & $\begin{array}{c}\text { Pruebas } \\
\text { utilizadas }\end{array}$ & $\begin{array}{c}\text { Porcentaje de } \\
\text { pruebas utilizadas }\end{array}$ & $\begin{array}{c}\text { Pruebas } \\
\text { faltantes }\end{array}$ \\
\hline Amazonas & 1.692 & 1.277 & 75 & 415 \\
Arauca & 1.484 & 1.250 & 84 & 234 \\
Boyacá & 168 & 168 & 100 & 0 \\
Cesar & 24 & 21 & 88 & 3 \\
Guainía & 1.128 & 452 & 40 & 676 \\
La Guajira & 3.504 & 2.237 & 64 & 1.267 \\
Nariño & 2.904 & 1.263 & 43 & 2641 \\
Norte de Santander & 792 & 523 & 66 & 269 \\
Putumayo & 16.684 & 13.931 & 83 & 16 \\
Vichada & 2904 & 478 & 69 & 2.753 \\
Total & 31.284 & 21.600 & & 9.426 \\
\hline
\end{tabular}


las necesidades reales de la zona intervenidas y el número de pruebas suministradas. De igual forma, se requiere de una logística adecuada en todos los niveles, lo que implica una gestión oportuna del envío de las pruebas, que garantice las condiciones de temperatura y humedad, tanto en el transporte como en el almacenamiento y en el lugar de uso.

El control de calidad debe formar parte integral de las estrategias de implementación de las pruebas de diagnóstico rápido en el país, para lo cual se requiere incluir el presupuesto necesario para actividades de control de calidad dirigidas tanto a las pruebas como al personal encargado del diagnóstico y el tratamiento (12).

El factor ambiental que más afecta la estabilidad de las pruebas es la temperatura, por lo que se debe procurar mantenerlas dentro del rango de temperatura sugerido por el fabricante (13). En un estudio previo en la provincia de Limpopo en Suráfrica, los autores recomendaban acciones que beneficien el desempeño y el uso, entre las cuales se encuentran almacenar las pruebas en ambientes que cuenten con aire acondicionado, evitar exponerlas a temperaturas superiores a $30{ }^{\circ} \mathrm{C}$, usar existencias con posible reposición dentro de las 24 horas siguientes a la solicitud, entrenar previamente al personal encargado de la atención al paciente y hacer el control de calidad (14).

El uso de las pruebas de diagnóstico rápido para malaria está regido por lineamientos de calidad establecidos por la Red Nacional de Laboratorios del Instituto Nacional de Salud, por lo que es importante que el personal encargado de hacerlo se encuentre activo en la red de control de calidad del diagnóstico de malaria, lo cual implica que en su respectivo nivel de referencia deben hacerse capacitaciones, evaluación del desempeño y supervisiones periódicas, para garantizar la calidad en el diagnóstico y el tratamiento de los pacientes. La supervisión periódica en los lugares de trabajo permite reconocer y resolver dificultades de manera oportuna, como también estimula la labor del trabajador de salud. En los casos particulares de los proyectos de intervención en los entes territoriales, cobra gran importancia garantizar la supervisión para posibilitar el cumplimiento de los objetivos y metas.

El control de calidad de las pruebas de diagnóstico rápido basado en la supervisión de las gotas gruesas es de interés para garantizar la buena atención del paciente. Sin embargo, no es viable hacerlo en todos los puntos de atención cuando se implementa una estrategia en un país (15), por lo que es necesario plantear este control de calidad en puestos centinela.

La variable logística, entendida como el transporte adecuado de las pruebas al sitio de aplicación de la estrategia, tiene relevancia debido a que es necesario garantizar de manera oportuna la ubicación de los insumos en el área de trabajo para evitar su vencimiento. A los entes territoriales, de acuerdo con las competencias que le son otorgadas por ley, les corresponde garantizar el transporte oportuno de los insumos, equipos o reactivos que les son adjudicados para el buen funcionamiento del programa. Esto puede ser viable cuando la coordinación de los proyectos de intervención ha realizado una gestión ordenada y clara con todos los niveles operativos.

Dentro del análisis de las variables con bajo cumplimiento en el ciclo de implementación, que no fueron clasificadas como críticas pero que pueden afectar el diagnóstico de la enfermedad, cabe resaltar el "control de calidad con paneles", el "cumplimiento de directrices de uso (población objeto) y la "encuesta de percepción de la comunidad frente a las pruebas de diagnóstico rápido".

El control de calidad con paneles permite contar con la vigilancia del funcionamiento de las pruebas en condiciones óptimas desde el momento de su adquisición hasta la fecha de su caducidad. Es necesario establecer y conocer que siempre que se realice una adquisición de pruebas de diagnóstico rápido a nivel nacional o adquisiciones grandes para distribuir a diferentes departamentos, se deben realizar acciones coordinadas con el Instituto Nacional de Salud para el adecuado desarrollo de la actividad. Esta institución, por liderar la red nacional de diagnóstico de malaria, debería contar con la capacidad de realizar o coordinar el control de calidad a los lotes de los estuches de pruebas de diagnóstico rápido bajo los lineamientos de la OMS (16). Sin embargo, entre las oportunidades de mejora es apropiado diseñar una estrategia que permita ajustar los protocolos de acuerdo con las últimas recomendaciones de la OMS. Dicho fortalecimiento se puede lograr mediante planes, programas y proyectos del nivel nacional, que cuenten con recursos nacionales e internacionales $(17,18)$.

Para un adecuado uso de las pruebas de diagnóstico rápido y un correcto aprovechamiento 
de los recursos, se debe tener presente que las pruebas deben ser aplicadas a la población ante la sospecha de la enfermedad, teniendo en cuenta las limitaciones propias y las indicaciones de uso. Es de gran interés establecer protocolos estandarizados de aplicación para todo el país, con el fin de evitar interpretaciones particulares por parte de los trabajadores de salud. Los protocolos deben incluir el tipo de conductas que se deben seguir, el diagnóstico diferencial, el control de calidad, la asistencia técnica y la supervisión (19).

De igual manera, para mejorar la aceptabilidad y facilitar las intervenciones de control de malaria con pruebas de diagnóstico rápido en las comunidades, es importante sensibilizar previamente a la población, especialmente a la indígena, proveyéndole información que posibilite el entendimiento de las acciones, para generar confianza y seguridad en las mismas.

La aplicación de las pruebas debe continuar viéndose como parte de una estrategia integral para el control de la malaria, en la cual es preciso considerar varios aspectos, tales como los epidemiológicos, ecológicos, sociales, operacionales y políticos, que permitan la sostenibilidad en el tiempo de las actividades propias del programa (19). Además, el uso de las pruebas de diagnóstico rápido debe entenderse como una alternativa complementaria en aquellas situaciones en las que la red de diagnóstico de microscopía se encuentre débil o cuando exista un desbordamiento de la capacidad diagnóstica (18).

Finalmente, teniendo en cuenta que la búsqueda activa en Colombia, dentro de los lineamientos nacionales, se encuentra indicada en situaciones de transmisión epidémica de malaria (3), se recomienda ampliar el concepto teniendo en cuenta las circunstancias planteadas por los departamentos intervenidos por el proyecto PAMAFRO.

En conclusión, el programa de Prevención y Control de ETV, la Red de Control de Calidad del Diagnóstico de Malaria y los proyectos de intervención dirigidos hacia los entes territoriales que consideren aplicar las pruebas de diagnóstico rápido para la malaria, deben procurar el fortalecimiento de las variables críticas expuestas en el presente trabajo.

Una de las limitaciones de la metodología utilizada para evaluar el ciclo de implementación, es que el análisis integral de las variables del ciclo de implementación de las pruebas depende del puntaje obtenido como variable crítica y del porcentaje de cumplimiento. Sin embargo, es necesario tomar en cuenta que la puntuación de cada indicador depende del nivel de satisfacción. Por lo tanto, para obtener puntajes altos en las variables, es necesario garantizar el cumplimiento de las actividades dentro de la estrategia.

En general, los resultados derivados de la información obtenida se ven limitados debido a la toma tardía de los datos, lo cual dificultó la recopilación, diligenciamiento de registros detallados y reconstrucción de indicadores de interés, impidiendo establecer una calificación más sensible.

\section{Agradecimientos}

Los autores expresan sus agradecimientos a: José Pablo Escobar, Consultor Nacional-OPS ETV; Roberto Montoya, consultor independiente en Enfermedades Tropicales.

ARubén Santiago Nicholls, asesor científico-técnico y a Liliana Jazmín Cortés y Olga Lucía Ospina, referentes de malaria, Grupo de Parasitología del Instituto Nacional de Salud, por sus aportes como expertos en el tema.

Al Grupo de Vigilancia en Salud Pública y Grupo de Almacén del Ministerio de la Protección Social, por proporcionar la información requerida para este estudio.

A los coordinadores de ETV y de los Laboratorios Departamentales de Salud Pública de Putumayo, Nariño, La Guajira, Amazonas, Norte de Santander, Guainía, Vichada, Arauca, Boyacá y Cesar, por su apoyo en la recolección de la información.

Finalmente, los autores agradecen el apoyo técnico y administrativo al grupo de PAMAFRO Colombia.

\section{Financiación}

Este trabajo fue financiado con recursos del Fondo Mundial para la Lucha contra el VIH, TBC y Malaria, a través del Organismo Andino de Salud - Convenio Hipólito Unanue (ORAS-CONHU), receptor principal del proyecto "Control de la malaria en las zonas fronterizas de la Región Andina: un enfoque comunitario" PAMAFRO.

\section{Conflicto de intereses}

Los autores declaran que durante las fases de la evaluación y análisis de este trabajo no incurrieron en conflictos de interés de ninguna naturaleza que hubieran podido afectar los resultados. 


\section{Referencias}

1. World Health Organization. A global strategy for malaria control. Geneva: WHO; 1993.

2. Warrell DA. Clinical features of malaria. En: Warrell DA, Gilles HM, editores. Essential malariology. 4 ed. London: Arnold; 2002. p.191-235.

3. Instituto Nacional de Salud. Protocolo de vigilancia de malaria. 2007. Fecha de consulta: 2 de diciembre de 2008. Disponible en: http://www.ins.gov.co/?idcategoria=5620\#.

4. López FJ, Schmunis G. Diagnóstico de malaria. Publicación científica 512. Washington, D.C.: OPS; 1988.

5. Moody A. Rapid diagnostic test for malaria parasites. Clin Microbiol Rev. 2002;15:66-78.

6. Organización Panamericana de la Salud. Investigación operacional sobre la implementación del uso de pruebas rápidas de diagnóstico de malaria. Guayaquil, 23-25 mayo 2005. OPS/DPC/CD/M/394/06. Geneva: WHO; 2005 Fecha de consulta: 2 de diciembre de 2008. Disponible en: http://www.paho.org/spanish/AD/DPC/CD/ravreda7-rdtsguayaquil.pdf.

7. Organización Mundial de la Salud. Uso de las pruebas rápidas en el diagnóstico rápido la malaria. Organización Mundial de la Salud. Oficina Regional para el Pacífico Occidental. Geneva: WHO; 2006. [Consultado: 7 de junio de 2009].Disponible en: http://www.wpro.who.int/NR/ rdonlyres/69975F00-AB92-4D21-8C1A-261F97938DF3/0/ Malaria_RDT_2ndEd_Spanish.pdf.

8. Organización Panamericana de la Salud. Investigación operacional sobre la implementación del uso de pruebas rápidas de diagnóstico de malaria: 2005 May. Report No.OPS/DPC/CD/M/394/06. Geneva: WHO; 2005.

9. García LA. Aplicación del análisis multicriterio en la evaluación de impactos ambientales (tesis). Barcelona: Universidad Politécnica de Catalunya; 2004. Fecha de consulta: 20 de junio de 2009. Disponible en: http://www. tdx.cat/TDX-0803104-125133.

10. Roche H, Vejo C. Análisis multicriterio en la toma de decisiones. Fecha de consulta: 12 de agosto de 2009. Disponible en: http://www.ccee.edu.uy/ensenian/catmetad/ material/MdA-Scoring-AHP.pdf
11. Ministerio de la Protección Social, Instituto Nacional de Salud. Malaria en La Guajira, balance 2007-2008 (hasta semana epidemiológica 34). Inf Quinc Epidemiol Nac. 2008;13:271-86.

12. World Health Organization. Malaria rapid diagnosis. Making it work. RS/2003/GE/05(PHL). Geneva: WHO; 2003. Fecha de consulta: 3 junio de 2009. Disponible en: http:// apps.who.int/malaria/cmc_upload/0/000/016/750/rdt2.pdf.

13. Ashley EA, Touabi M, Ahrer M, Hutagalung R, Htun $\mathbf{K}$, Luchavez $\mathbf{J}$, et al. Evaluation of three parasite lactate dehydrogenase-based rapid diagnostic tests for the diagnosis of falciparum and vivax malaria. Malar J. 2009;8:241.

14. Moonasar D, Goga AE, Frean J, Krugger $\mathbf{P}$, Chandramohan D. An exploratory study of factors that affect the performance and usage of rapid diagnostic tests of malaria in the Limpopo Province, South Africa. Malar J. 2007;6:74.

15. McMorrow ML, Masania MI, Kahigwa E, Abdulla SM, Kachur SP. Quality assurance of rapid diagnostic tests for malaria in routine patient care in rural Tanzania. Am J Trop Med Hyg. 2010; 82:151-5.

16. Organización Mundial de la Salud. Towards quality testing of malaria rapid diagnostic test: evidence and methods. Geneva: WHO; 2006. Fecha de consulta: 18 de junio de 2009. Disponible en: http://www.wpro.who.int/NR/ rdonlyres/89F9DB09-9BE2-4659-AE08-FE84195FEDBA/0/ web3_QARDTreport.pdf.

17. World Health Organization. Methods manual for laboratory quality control testing of malaria rapid diagnostic tests. Geneva: WHO; 2008. Fecha de consulta: 5 de junio de 2009. Disponible en: http://www.wpro. who.int/NR/rdonlyres/A31142C7-CA46-465F-83D7776C691C79BA/0/RDTLaboratoryQCtestingMethManuV5 _a_WEBVERSIONAug2008.pdf.

18. Chandler $\mathbf{C l}$, Whitty $\mathbf{C J}$, Ansah EK. How can malaria rapid diagnostic tests achieve their potential? A qualitative study of a trial at health facilities in Ghana. Malar J. 2010;9:95.

19. World Health Organization. Achievements problems \& strategies. Malaria control. WHO/MAL/99.1087. Geneva: WHO; 1999. 